\title{
Critique des fondements et usages de l'écosystémie dans le domaine du partenariat école-famille-communauté : application d'une perspective contextuelle et socioculturelle dans le cadre du programme Famille, école, communauté, réussir ensemble
}

\author{
Dany Boulanger, François Larose, Serge J. Larivée, Yves Couturier, Corinne \\ Mérini, François Blain, Valérie Cusson, Daniel Moreau et Naomi Grenier
}

Volume 57, numéro 2, 2011

URI : https://id.erudit.org/iderudit/1006300ar

DOI : https://doi.org/10.7202/1006300ar

Aller au sommaire du numéro

Éditeur(s)

École de service social de l’Université Laval

ISSN

1708-1734 (numérique)

Découvrir la revue

Citer cet article

Boulanger, D., Larose, F., Larivée, S. J., Couturier, Y., Mérini, C., Blain, F., Cusson, V., Moreau, D. \& Grenier, N. (2011). Critique des fondements et usages de l'écosystémie dans le domaine du partenariat école-famille-communauté : application d'une perspective contextuelle et socioculturelle dans le cadre du programme Famille, école, communauté, réussir ensemble. Service social, 57(2), 129-157. https://doi.org/10.7202/1006300ar
Résumé de l'article

Cet article s'inscrit au coeur du débat sur la nature du partenariat école-famille-communauté et sur ses logiques sous-jacentes. Nous y contribuons en questionnant les fondements et les usages de l'écosystémie qui est, en principe, à la base des pratiques collaboratives et de l'engagement parental. En situant l'écosystémie au regard de la notion de métathéorie, nous inscrirons les pratiques collaboratives sur un continuum qui reflète la nature et le degré d'interaction entre les acteurs. Nous prenons position au chapitre du partenariat école-famille-communauté en adoptant une perspective écosystémique d'orientations contextuelle et socioculturelle. Nous nous appuierons sur le programme Famille, école, communauté, réussir ensemble (FECRE), mis en oeuvre au Québec entre 2002 et 2009, pour illustrer

l'actualisation d'une telle logique écosystémique dans le cadre de pratiques collaboratives ainsi que de l'engagement parental. 


\title{
Critique des fondements et usages de l'écosystémie dans le domaine du partenariat école-famille-communauté : application d'une perspective contextuelle et socioculturelle dans le cadre du programme Famille, école, communauté, réussir ensemble
}

\author{
Dany Boulanger \\ François Larose \\ Serge J. Larivée \\ Yves Couturier \\ Corinne Mérini \\ François Blain \\ Valérie Cusson \\ Daniel Moreau \\ Naomi Grenier
}

\section{RÉSUMÉ}

Cet article s'inscrit au cœur du débat sur la nature du partenariat école-famille-communauté et sur ses logiques sous-jacentes. Nous y contribuons en questionnant les fondements et les usages de l'écosystémie qui est, en principe, à la base des pratiques collaboratives et de l'engagement parental. En situant l'écosystémie au regard de la notion de métathéorie, nous inscrirons les pratiques collaboratives sur un continuum qui reflète la nature et le degré d'interaction entre les acteurs. Nous prenons position au chapitre du partenariat école-famillecommunauté en adoptant une perspective écosystémique d'orientations contextuelle et socioculturelle. Nous nous appuierons sur le programme Famille, école, communauté, réussir ensemble (FECRE), mis en œuvre au Québec entre 2002 et 2009, pour illustrer l'actualisation d'une telle logique écosystémique dans le cadre de pratiques collaboratives ainsi que de l'engagement parental.

Mots clés : Partenariat école-famille-communauté; pratiques collaboratives; engagement parental; écosystémie; métathéorie; théories de l'activité; dynamic system theory; interculturalité

\begin{abstract}
In this article, we explore the heart of the debate on the nature of the school-family-community partnership and its underlying rationales. We challenge the underpinnings and uses of the ecosystem which is considered at the root of collaborative practices and parental engagement. By inserting the ecosystem into a metatheory, we place collaborative practices along a continuum that reflects the nature and the degree of interactions between those engaged. Our standpoint looks at this partnership in relation to an ecosystemic perspective that is based on contextual and sociocultural perspective. To illustrate how such an ecosystemic logic can be applied to collaborative practices and parental engagement, we refer to the program Famille, école, communauté, réussir ensemble (FECRE), which was implemented in the province of Quebec between 2002 and 2009.
\end{abstract}

Keywords: School, family; community partnership; collaborative practices; parents' engagement; ecosystemy; meta-theory; activity theory; dynamic system theory; interculturality 


\section{INTRODUCTION}

Cet article se situe dans le contexte de l'émergence, au Québec, de plusieurs projets visant à favoriser l'établissement d'un partenariat entre l'école, la famille et la communauté. Bien que les discours scientifiques et ministériels promeuvent la construction de liens multilatéraux entre les acteurs, les pratiques collaboratives et les conditions d'actualisation de l'engagement parental s'inscrivent peu dans une telle logique écosystémique. Afin de contribuer au débat actuel sur la nature du partenariat école-famille-communauté et sur ses logiques sous-jacentes, nous questionnons les fondements et les usages de l'écosystémie dans ce domaine de recherche. En nous appuyant sur Larivée, Kalubi et Terrisse (2006), nous proposons de situer les pratiques collaboratives sur un continuum en fonction du degré d'interaction entre les acteurs et d'intégration entre les diverses composantes de l'écosystème, en particulier les objets culturels. Après avoir critiqué la perspective mécaniste dominante dans ce domaine, nous proposons de définir le partenariat école-famille-communauté au regard d'une perspective écosystémique d'orientations contextuelle et socioculturelle. Nous analyserons rapidement le programme Famille, école, communauté, réussir ensemble (FECRE), mis en œuvre au Québec entre 2002 et 2009, pour illustrer l'actualisation d'une telle logique écosystémique dans le cadre des pratiques collaboratives ainsi que de l'engagement parental.

\section{Le contexte de mise en œuvre d'un partenariat entre l'école, la famille et la communauté}

L'entente de complémentarité des services entérinée en 2003 entre les réseaux québécois de la santé et des services sociaux ainsi que celui de l'éducation réaffirme la nécessité d'articuler l'action des divers acteurs intervenant au sein de ces secteurs (Gouvernement du Québec, 2003a). La réussite éducative y est considérée comme une visée partagée par les professionnels de l'intervention éducative et socioéducative œuvrant tant dans l'espace scolaire qu'en dehors de ce dernier (Gouvernement du Québec, 2008). Les parents y sont aussi désignés comme des acteurs centraux. Dans le contexte actuel, les intervenants socioéducatifs tels que les travailleurs sociaux, mais aussi de nombreux praticiens des métiers relationnels, sont de plus en plus appelés à situer leur champ d'action dans l'univers scolaire (Couturier, Larose et Bédard, 2009; Larose, Lenoir, Couturier et Bédard, 2011sp). En fait foi la multiplication des pratiques intersectorielles et interprofessionnelles mobilisant les intervenants de l'institution scolaire et des réseaux extra-scolaires autour de la réussite éducative (Greenberg, Feinberg, Meyer-Chilenski, Spoth et Redmond, 2007; Lemay, Giguère et Marchand, 2008).

L'articulation entre ces intervenants fait appel à la contribution de l'ensemble des ressources du milieu (Baum, 2002). Dans cette veine, les discours ministériels promeuvent la reconnaissance des parents, toutes classes sociales confondues, en tant que partenaires de l'école et du milieu (Gouvernement du Québec, 2003b, 2004). Les pratiques actuelles s'y inscrivent au cœur d'un champ en émergence au Québec, celui du partenariat école-famillecommunauté (Gouvernement du Québec, 2008).

Ce domaine de recherche est très peu circonscrit aux plans conceptuel, théorique et épistémologique (Price-Mitchell, 2009). Les « construits » qui y sont véhiculés sont peu définis et ils sont le plus souvent désignés comme des synonymes (Fan et Chen, 2001). De plus, ils 
s'avèrent peu situés théoriquement (Boethel, 2003). II apparaît alors important de nous positionner à cet égard. Nous définissons le partenariat école-famille-communauté comme une action (Mérini, 2001) de nature dynamique (Moll, 1993; Moll, Amanti, Neff et Gonzalez, 1992) et à visée intégratrice (De Gioia, 2009) réalisée par un ensemble d'acteurs à la charnière de plusieurs milieux ou systèmes sociaux (Fleer et Williams-Kennedy, 2001). Bien que sollicitant le terme de partenariat parce qu'il est considéré, d'un point de vue disciplinaire ${ }^{1}$, comme représentatif du domaine à l'étude (Mérini, 2001; Driessen, Smit et Sleegers, 2005), nous désignons les pratiques comme collaboratives ${ }^{2}$. À l'instar de Larivée, Kalubi et Terrisse (2006), nous considérons plus ou moins partenariales les pratiques collaboratives, en fonction du degré d'engagement des parents ainsi que du degré de relation et de consensus entre les acteurs. Pour nous, les pratiques collaboratives désignent un processus (Sykes, 2001) d'intégration et d'articulation entre les objets véhiculés dans et par les différentes cultures auxquelles s'expose l'enfant (Mérini, 1995; Hughes et Greenhough, 2007), processus faisant appel à une négociation entre les acteurs (Christenson et Sheridan, 2001; Landry, Levin, Rowe et Nickelson, 2010) cherchant à établir une forme de consensus (Larivée, Kalubi et Terrisse, 2006). La notion d'engagement traduit l'intégration de la personne à son milieu et son appartenance socioculturelle (Levasseur, Richard, Gauvin et Raymond, 2010). Selon ces auteurs, l'engagement caractérise un degré élevé d'investissement de la part de l'individu et il s'exerce dans le cadre de pratiques ou d'activités sociales. Considéré comme un acteur, l'individu engagé construit son rapport à la culture et il interagit avec des tiers sur une base réciproque (Ferlazzo, 2011; Nagda, McCoy et Barrett, 2006). L'engagement passe alors par une action de négociation et de co-construction sociale et il s'exerce au cœur d'interfaces sociales (Price-Mitchell, 2009; Weerts et Sandmann, 2010).

Comme nous le démontrerons ultérieurement, de telles définitions peuvent s'appliquer au programme Famille, école, communauté, réussir ensemble (FECRE), mis en œuvre au Québec entre 2002 et 2009. Le programme cadre de façon toute particulière avec les fondements écosystémiques véhiculés dans le domaine du partenariat école-famille-communauté, et ce au Québec et ailleurs. Bien que possédant ses spécificités, il s'inscrirait dans la lignée de projets se revendiquant de cette perspective, tels qu'on les retrouve particulièrement aux États-Unis. C'est en raison, notamment, de la logique anglo-saxone à la base de plusieurs projets québécois et de la référence qui est faite, dans FECRE, à Bronfenbrenner et à Epstein ${ }^{3}$, qui

1. Plusieurs indices montrent que le partenariat correspond à un champ disciplinaire ou du moins à un secteur de recherche articulé autour d'un certain corpus de savoirs, contrairement à la collaboration. Le partenariat écolefamille-communauté serait un domaine particulier du champ du partenariat.

2. Que ce soit dans le domaine du partenariat école-famille-communauté ou encore dans celui de l'interprofessionnalité, la documentation scientifique renvoie très peu à la notion de pratique partenariale. Dans le domaine du partenariat école-famille-communauté, les auteurs se réfèrent très généralement aux pratiques « de nature » partenariale ou « inscrites dans un contexte » partenarial. Les pratiques interprofessionnelles sont considérées comme des pratiques collaboratives lorsque l'interaction s'exerce sur une base réciproque et qu'elle vise à engager de façon active la population ciblée (Mickan, Hoffman et Nasmith, 2010). Nous trouvons alors pertinent de nous appuyer sur cette acception comme base de conceptualisation des pratiques dans le domaine du partenariat école-famille-communauté.

3. Dans le cadre de référence de FECRE, il est fait mention de ces auteurs, mais nous situerons ce programme au regard d'une autre perspective théorique. Ce seraient essentiellement les logiques d'usage des modèles théoriques identifiés dans le cadre de référence ainsi que les métathéories dans lesquelles s'inscrivent ces modèles qui expliquent l'apparence d'un écart entre la perspective annoncée et celle traduite dans la réalité. Par 
constituent des références majeures aux États-Unis, que nous le situons au cœur d'un vaste mouvement en faveur d'un partenariat à établir entre l'école, la famille et la communauté (Davies et Guppy, 1997; Gouvernement du Québec, 2003b; Lenoir, 2005; Meuret, 2006). Au Québec, FECRE prend particulièrement ancrage dans le contexte d'émergence de projets, de mesures, d'approches, d'initiatives et de programmes à base partenariale depuis le début des années 2000, pratiques qui s'appuient en principe sur une logique écosystémique (Larose, Bédard, Couturier, Larivée, Lenoir, Lenoir et Terrisse, 2010). Celle-ci fonde toute action ultimement destinée à l'enfant sur l'interaction entre les acteurs qui font office de figures significatives pour celui-ci (Bronfenbrenner, 1979). L'influence potentielle des pratiques sur l'enfant découle de leur caractère «partenarial » et de leur finalité de soutien à l'engagement parental (Bronfenbrenner, 1986).

Dans une telle perspective, les pratiques doivent se déployer collectivement et ainsi faire appel à la mobilisation d'un ensemble d'acteurs professionnels et non professionnels (les parents) dans une démarche commune (Deschesnes, Martin et Hill, 2003). Suivant Selsky (1991), nous entendons par mobilisation une forme d'action collective visant à répondre à un but commun, acception proche de celle donnée au partenariat (Henderson et Mapp, 2002). Tyler (1996) et Christenson (2003) considèrent que les pratiques fondées à la fois sur une logique écosystémique et «partenariale » doivent s'exercer dans un contexte relationnel égalitaire et se traduire par un dialogue ouvert et émergent entre l'ensemble des acteurs. Les parents de milieux socio-économiquement faibles (msef) doivent être activement engagés au plan scolaire. De plus, les projets dans lesquels s'insèrent ces pratiques doivent permettre la structuration de l'action «partenariale » ou «collaborative » en fournissant, aux différents acteurs, des dispositifs soutenant de façon effective la mobilisation et l'engagement.

Bien que se réclamant d'une perspective écosystémique, les pratiques qui se réalisent dans le cadre de tels projets s'exercent le plus souvent sur une base relationnelle inégalitaire et hiérarchique et elles favorisent peu la mobilisation et l'engagement des intervenants du milieu ainsi que des parents de msef (Lott, 2001). Les pratiques actuelles renforceraient l'écart entre l'école, la famille et la communauté plutôt que de créer, de façon effective, des conditions de rapprochement entre ces univers éducatifs (Wilgus, 2005). Ce phénomène tend à perdurer malgré l'implantation massive de mesures dans de nombreuses écoles de msef et l'évolution des connaissances scientifiques en matière de partenariat école-famille-communauté (Fan et Chen, 2001). Bien que les auteurs réaffirment les principes au fondement de ce partenariat en insistant particulièrement sur l'égalité, la mutualité ainsi que la réciprocité entre les acteurs (Keyes, 2002; Powell, 2001), les caractéristiques énoncées demeureraient de l'ordre du discours et ne se traduiraient pas par des changements de pratiques (Jordan, Orozco et Averett, 2001). Durant la dernière décennie, certains auteurs proposent de nouveaux cadres d'analyse et modèles de pratique en s'appuyant sur une mise en question relative des fondements écosystémiques du partenariat école-famille-communauté tout en prenant position au plan épistémologique (De Gioia, 2009; Hughes et Greenhough, 2007; Price-Mitchell, 2009).

ailleurs, tout rapport au réel implique une réflexion sur la mise en action des savoirs et donc sur la construction d'objets pouvant être réifiés. Cette dimension sera véhiculée dans l'article par la référence que nous ferons au caractère structural des pratiques et à l'organisation des principes théoriques autour de métathéories. Ces éléments seront précisés ultérieurement. 
Ces auteurs invitent les lecteurs à approfondir cet exercice en se penchant plus explicitement sur les fondements et les logiques d'usage de l'écosystémie dans ce domaine, ceci afin d'identifier les ressorts du partenariat, autrement dit, les processus et dynamiques qui animent et structurent les pratiques.

\section{L'écosystémie : principes et fondements}

L'écosystémie désigne un vaste champ de recherche. Sa trajectoire plurielle est marquée par des migrations disciplinaires multiples entre des champs aussi variés que l'anthropologie, la sociologie, l'administration, l'ingénierie, la psychologie, le service social et les sciences de l'éducation (Gibbs, 1979; Amatea Ellens et Sherrard Peter, 1994; Ungar, 2002). L'écosystémie est donc teintée par plusieurs « courants » théoriques et logiques épistémologiques. Ceci a l'avantage d'en faire un « paradigme » de référence générique qui se décline en un ensemble d'approches et de modèles et qui se prête à différentes perspectives épistémologiques. Par ailleurs, cette même caractéristique a le défaut de la constituer comme une « coquille vide » parfois dénuée de sens, ceci en raison, particulièrement, du rapport restreint et problématique qu'entretiennent les auteurs avec ses fondements (Kondrat, 2002; Sontag, 1996). La nature des fondements et du rapport qu'y entretiennent les chercheurs a des incidences sur la pratique des divers intervenants. Les limites identifiées, dont les bases épistémologiques (métathéoriques plus particulièrement) seront reprises ultérieurement, justifient la clarification des origines disciplinaires de l'écosystémie

Deux repères stables permettent de circonscrire les origines de l'écosystémie. S'ils ne rendent pas compte de la complexité constitutive de l'écosystémie, ils permettent de la situer autour des principaux courants qui l'ont constituée. D'abord, elle naît au croisement de l'écologie et de la théorie des systèmes (Tessier, 1989; Tessier et Tarabulsy, 1996; Terrisse et Larose, 2003). Ensuite, c'est principalement autour des théories du développement de l'enfant que se situent les travaux des fondateurs de l'écosystémie. À cet égard, nous pensons particulièrement à Kelly (1966), Garbarino et Sherman (1980), Sameroff (1983), Lerner (1998, 2004) et Bronfenbrenner ${ }^{4}(1974,1977,1979,1986,2005)^{5}$.

De manière générique, l'écosystémie se caractérise par le postulat d'un lien récursif entre l'individu et son environnement. Cet environnement fournit à la personne des contraintes, des ressources et des potentialités. L'individu construit activement son rapport au monde, en

4. Bronfenbrenner a créé son premier modèle écosystémique en 1979. II a développé le modèle bioécologique au début des années 1990. Dans le premier cas, l'accent est mis sur les principes organisateurs de l'écosystème en référence à une logique de cohérence et, sous cet angle, les liens sociaux sont essentiellement considérés comme fonctionnels, en dépit de la posture phénoménologique qu'il annonce au début de son ouvrage de 1979. À cet égard, le lecteur peut se référer à Branco (1996), Henrich (2006), Tudge, Gray et Hogan (1997), Overton (1998) et Wong (2001). Son deuxième modèle aborde l'interaction dans une perspective contextuelle, mais l'analyse de cette interaction se limite au microsystème. Malgré ces grands traits, la description de ces modèles mérite de plus amples explications, notamment aux plans épistémologique et épistémique.

5. Ce n'est pas là un portrait complet, il s'agit des principaux auteurs ayant contribué aux théories du développement de l'enfant sous l'angle de l'écosystémie. À titre de référence, nous vous référons aussi à Gibson (1962, 1967), qui s'est intéressé aux perceptions, à Albee (1982), dont les travaux semblent avoir influencé ceux de Kelly, et à Baltes (1987), qui renvoie à l'évolution de la personne sous l'angle des phases de discontinuité qu'elle rencontre. Lerner s'est notamment appuyé sur Baltes. Finalement, nous identifions Luhmann (2006) qui définit, en termes simples, le système par rapport à ce qui le distingue. 
s'appropriant contextuellement les objets qui y sont véhiculés, en y sollicitant et y construisant des ressources (facteurs de protection, notamment) et en prenant les facteurs de risque comme des occasions de défi optimal (Lerner, 2006; Riegel, 1976). Bien sûr, la nature du rapport entre l'individu et l'environnement varie selon la perspective épistémologique et théorique du chercheur, mais l'ensemble des auteurs semble s'entendre sur la présence d'un lien bilatéral entre ces deux entités. La personne construit activement son rapport à l'environnement. La dimension d'interaction est centrale dans un tel processus, c'est en fait en interagissant entre eux sur une base à la fois contextuelle et stable que les acteurs peuvent soutenir l'évolution de leur environnement et contribuer à leur culture (Cole, 1999; Lerner, Freund, De Stefanis et Habermas, 2001). Les personnes établissent des échanges au sein et à la charnière des systèmes qu'elles fréquentent et, plus largement, de l'ensemble des systèmes composant leur environnement social. Cet environnement possède une configuration particulière qui en traduit la structure et les fonctionnalités de l'interaction entre les systèmes le composant (Overton, 1998). Nous préférons parler d'écosystème, ce dernier étant caractérisé par les composantes interactionnelle, socioculturelle ${ }^{6}$ et épistémique de l'environnement (Sameroff, 1983).

Nous nous appuyons sur le modèle de Bronfenbrenner (1979) pour caractériser l'écosystème et ainsi en identifier les paramètres organisateurs et structurants. C'est généralement en référence à ce modèle que les auteurs caractérisent l'écosystème. Pour Bronfenbrenner, ce dernier prend la forme de systèmes sociaux emboîtés les uns dans les autres, un peu à l'image des poupées russes. II s'agit en quelque sorte de « niveaux systémiques » situés à des échelons hiérarchiques distincts. Bronfenbrenner fait référence à l'ontosystème, au microsystème, au mésosystème, à l'exosystème et au macrosystème. L'ontosystème représente l'enfant lui-même avec ses caractéristiques. Le microsystème est celui dans lequel participe directement l'enfant, soit la famille ou l'école par exemple. Ce système est caractérisé par les activités réalisées par l'enfant, par les rôles des adultes et par la relation enfant-adulte. Le mésosystème désigne le lien entre deux microsystèmes. II s'agit de l'interaction entre des acteurs, les parents et personnels enseignants par exemple. Cette interaction peut aussi se manifester de façon indirecte, par le biais de processus ou de dynamiques de médiation. Nous reviendrons ultérieurement sur cette considération. Dans le cadre de cet article, l'analyse des pratiques se situera au niveau du mésosystème. L'exosystème caractérise le système auquel ne participe pas directement l'individu mais dont il subit l'influence, pensons par exemple à ce que représente la commission scolaire pour le parent qui n'y est pas engagé. Finalement, l'auteur réfère au macrosystème pour désigner les normes, les idéologies, les discours, les valeurs, les savoirs et les représentations dominantes d'une collectivité.

Bronfenbrenner (1979) fournit une modélisation de l'écosystème qui s'avère utile pour réfléchir aux activités, aux pratiques et aux interactions sociales dans une logique multidimensionnelle. Or, comme c'est le cas pour tout modèle, les principes qu'il propose n'ont

6. Dans le cadre de cet article, nous traitons de culture au sens d'un ensemble d'outils socio-sémantiques et relationnels et de pratiques socioculturelles dont la fonction est de structurer et de médier le rapport qu'entretient l'individu avec son environnement (Cole, 1999; Valsiner, 2003, 2009). Elle constitue par ailleurs une composante de l'écosystème (Sameroff, 1983). 
de sens qu'à la lumière des fondements épistémologiques et métathéoriques dans lesquels ils sont situés (Overton, 2007). Une métathéorie agit un peu comme une épistémologie, mais elle se situe à un niveau heuristique plus abstrait. Sous l'angle métathéorique, les théories, les approches et les modèles, qui sont à la base de l'intervention, s'articulent autour d'une conception particulière de l'écosystème et donc de l'interaction, du lien individu-environnement et de la dimension de culture (Overton, 1998). Ainsi, le principe de l'interrelation entre les systèmes, véhiculé par Bronfenbrenner (1979) notamment, prend un sens différent selon la perspective métathéorique adoptée par le chercheur. Parallèlement, celle-ci façonne les pratiques à base collective, elle en détermine les orientations, les finalités et les composantes (Idem).

En lien avec les travaux réalisés par Reese et Overton $(1970)^{7}$, la documentation scientifique renvoie généralement à trois métathéories : mécaniste, organiste et contextuelle ${ }^{8}$ (Morris, 1997; Sameroff, 1983; Thomas, 1996; Witherington, 2007). Le dialectisme est associé au contextualisme; il s'inscrit plus particulièrement en extension de celui-ci (Overton, 1998). Nous assistons à l'émergence de perspectives intermédiaires constituées de l'amalgame des principes de plusieurs métathéories, pensons particulièrement au courant organiste-contextuel qui intègre fortement la composante socioculturelle et qui s'inscrit dans une logique dialectique (Overton, 2007; Overton et Ennis, 2006). Nous parlerons d'un courant contextuel et socioculturel pour le désigner. Les différentes métathéories ${ }^{9}$ reflètent des représentations particulières des conditions d'intégration et d'interaction entre les systèmes. Ceci est particulièrement mis en évidence par Overton (1998) ainsi que par Wapner et Demick (1998).

Le lien entre les systèmes sociaux (mésosystème) peut ainsi être lu à travers des perspectives variées. Les microsystèmes peuvent être représentés de façon plus ou moins cloisonnée ou, au contraire, plus ou moins intégrée. Ils peuvent simplement être considérés comme associés entre eux, ils peuvent interagir ou encore s'articuler de manière dynamique. Tout dépend de la métathéorie sollicitée. Telles que nous les avons définies, les pratiques collaboratives s'exercent dans une logique plus ou moins partenariale selon la nature et le degré d'interaction et d'intégration entre les acteurs. La formation d'un consensus reflète une forme d'articulation croisée des objets culturels véhiculés par les différents acteurs, contrairement à l'application d'une logique additive caractérisée par le partage superficiel de points de vue. Nous pouvons situer les pratiques sur un continuum en fonction des caractéristiques qui viennent d'être mentionnées et en fonction des conditions de mise en œuvre de l'engagement parental. Les paramètres interactionnels du partenariat école-famillecommunauté dépendent de la perspective écosystémique et donc de la lecture du mésosystème qu'elle autorise.

7. Ils se sont en partie et pour certaines considérations appuyés sur Pepper (1942).

8. Ce n'est pas l'objet de ce texte de définir et de différencier ces métathéories. Des éléments de définition sont intégrés à l'analyse du partenariat. Nous référons le lecteur à Overton (1998), ainsi qu'à Wapner et Demick (1998) pour une définition de ces métathéories.

9. Les métathéories mères et celles qui se sont construites à partir de l'intégration des principes de deux métathéories. 


\section{Le partenariat école-famille-communauté dans une perspective mécaniste}

Plusieurs auteurs considèrent que le domaine du partenariat école-famille-communauté s'ancre dans une perspective mécaniste et en est fortement teinté, et ce en dépit des discours scientifiques ou gouvernementaux qui prônent l'établissement d'une articulation complexe à établir entre les systèmes (Boethel, 2003; Lawson, 2003; Edwards et Warin, 1999; Nichols, 2007; De Gioia, 2009; Souto-Manning et Swick, 2006). En témoigne la prédominance des logiques déficitaire et compensatoire à la base des pratiques collaboratives ainsi que des conditions de réalisation de l'engagement parental (Price-Mitchell, 2009). Pour cette auteure, le lien entre les systèmes (mésosystème) est représenté de manière hiérarchique, statique et il s'établit en premier lieu autour de la culture scolaire. Celle-ci constitue la norme en matière de partenariat école-famille-communauté, c'est autour d'elle que se structurent les pratiques collaboratives (Souto-Manning et Swick, 2006). Ces dernières seraient avant tout l'affaire des personnels enseignants et, en partie, des directions d'école qui en assument le leadership, qui les définissent et les réalisent en fonction de leur propre logique d'action. Les besoins et les intérêts des intervenants du milieu sont souvent négligés au profit de finalités scolaires et le développement communautaire constitue une visée secondaire, sinon absente des pratiques collaboratives (McClenaghan, 2000; Cantillon, 2006). Par ailleurs, les compétences éducatives parentales sont peu reconnues par les personnels scolaires, les pratiques éducatives parentales étant jugées à risque pour l'enfant, particulièrement lorsqu'elles se déploient au sein de populations vulnérables (Boudreault, Kalubi, Sorel, Beaupré et Bouchard, 1998; Boudreault, Kalubi et Moreau, 2000).

Si le discours scientifique promeut l'adoption d'une vision commune et le partage d'un but entre les «acteurs» (Henderson et Mapp, 2002), cette vision et ce but sont le plus souvent prédéterminés en fonction des orientations, des finalités et des intérêts qui sont ceux des personnels scolaires (Lawson, 2003; Vincent et Martin, 2002). Conformément à une logique mécaniste, l'homogénéité des pratiques et des formes de pensée s'établissant autour de la culture scolaire est privilégiée et non pas la prise en compte de la diversité des points de vue. La culture scolaire faisant office de norme à l'interaction, l'ensemble des acteurs de msef, particulièrement, doit dans une certaine mesure «servir » la mission de l'école et en partie y adhérer de façon à faciliter le transfert unidirectionnel de savoirs de nature scolaire de l'école à la maison et ce, en agissant à la périphérie de l'institution scolaire. Nous nous appuyons ici sur Vincent (1993) ainsi que sur Vincent et Tomlinson (1997) qui constatent le rapport d'extériorité qu'entretiennent les intervenants du milieu ainsi que les parents de msef à l'égard de l'école et leur rôle d'agents de soutien vis-à-vis des pratiques pédagogiques (plus largement de la culture scolaire). Pour ces mêmes auteurs, les pratiques collaboratives exercent, d'une part, une fonction de maintien des intervenants de la communauté ainsi que des parents de msef à la périphérie de l'école et, d'autre part, une fonction de reproduction des rapports hiérarchiques que ceux-ci entretiennent vis-à-vis des personnels scolaires.

La prédominance d'une perspective mécaniste dans le domaine du partenariat école-famillecommunauté se décèle aussi par le caractère peu intégratif et structuré des pratiques collaboratives ainsi que des conditions de mise en œuvre de l'engagement parental. Les projets à caractère partenarial ne comportent généralement pas de dispositifs facilitant et soutenant de 
façon effective la mobilisation des diverses catégories d'acteurs (Breitborde et Swiniarski, 2002). Parallèlement, l'interprofessionnalité constituerait rarement une composante des projets et des pratiques, sauf dans le cadre de quelques mesures déployées notamment au Québec et comportant une forte dimension communautaire.

Les écrits scientifiques renvoient peu aux dimensions structurantes, structurelles et structurales des pratiques collaboratives (McGarth, 2007). Ces dernières prennent généralement la forme d'un ensemble d'activités disparates ${ }^{10}$ (peu liées ou articulées entre elles) qui s'actualisent sur une base situationnelle en fonction des besoins des personnels enseignants. Ceux-ci sollicitent généralement les parents de msef lorsque l'enfant présente des difficultés d'adaptation sociale et scolaire (Lawson, 2003). L'engagement parental constitue l'objet de ces activités ainsi que l'objet du discours des personnels enseignants et non pas une composante des pratiques. C'est en ce sens qu'Eccles et Harold (1993) soulignent le caractère peu intégratif et stable des pratiques et que Vincent (1993) souligne, comme nous le mentionnions, le rapport d'extériorité qu'entretiennent les parents de msef à l'égard de l'école.

D'un point de vue métathéorique, nous pouvons affirmer que le partenariat école-famillecommunauté est peu propice au rapprochement entre les univers éducatifs, un principe fortement promu par Epstein $(1987,1995,2005)$ aux États-Unis et par Deslandes et ses collègues (Deslandes et Lafortune, 2001; Deslandes, Royer, Potvin et Leclerc, 1999) au Québec. Parallèlement, le fait que les pratiques collaboratives s'organisent et se structurent autour d'une logique compensatoire reflète et participe au cloisonnement des systèmes ainsi qu'au maintien du phénomène de discontinuité culturelle qui est à la base de l'échec scolaire des enfants de msef (Lawson, 2003; Wilgus, 2005). En nous appuyant sur la typologie élaborée par Larivée, Kalubi et Terrisse (2006) qui constitue l'une des bases de la définition des pratiques et de l'engagement présentée préalablement, il s'avère que les pratiques collaboratives s'inscrivent généralement dans un registre peu partenarial. Elles sont peu propices à l'établissement de relations soutenues et inscrites dans une certaine proximité ${ }^{11}$ entre les différents acteurs professionnels et non professionnels. Les pratiques collaboratives s'articulant à la culture scolaire, elles favorisent peu l'intégration croisée des différents points de vue et des compétences de ces acteurs et, conséquemment, elles ne peuvent aboutir à un consensus (Fitzgerald et Goncü, 1993). Elles permettent peu l'articulation des différentes cultures (Cairney, 2001), c'est-à-dire la mise en œuvre d'un processus d'interculturalité (Mérini, 1995). Parallèlement, le parent est peu engagé, sinon en marge de l'école (Lawson, 2003).

10. À la base de la planification et de l'évaluation de projets se revendiquant d'une logique partenariale, nous retrouvons des typologies de l'engagement parental. Celles-ci situent l'engagement autour de types ou de formes spécifiques. Les projets ne comporteraient généralement pas de dispositifs permettant d'articuler entre elles les différentes formes d'engagement et, parallèlement, les différents types d'engagement sont la plupart du temps évalués autour de modèles unidimensionnels (Christenson, 2003, Fan et Chen, 2001; Jeynes, 2005; Sheldon et Van Voorhis, 2004). Or, l'adoption d'une perspective écosystémique intégratrice nécessite de circonscrire les modalités d'articulation entre les différents registres et formes d'action sociale, de façon à identifier, par exemple, en quoi l'engagement du parent à l'école supporte et médie son engagement à la maison.

11. Proximité au sens d'une relation favorable à l'intégration croisée des savoirs et des pratiques. 
Beaucoup d'auteurs se revendiquant d'une perspective écosystémique et se référant parfois au caractère intégratif, contextuel et dynamique de l'écosystème glissent dans une logique mécaniste, fondant ainsi les pratiques sur une perspective déficitaire ou compensatoire (Lott, 2001; Souto-Manning et Swick, 2006). Ce phénomène s'explique fortement par le rapport restreint aux fondements de l'écosystémie chez les auteurs et par l'inscription de modèles ou d'approches écosystémiques dans un ensemble de logiques peu compatibles entre elles (PriceMitchell, 2009). Cette tendance se constate plus largement dans le domaine de l'intervention éducative et socioéducative (Sontag, 1996). L'économie des fondements se traduit généralement par un usage limité et erroné des principes véhiculés par les approches et les modèles et, conséquemment, par des glissements dans la façon de concevoir et d'analyser les pratiques à base collective (Boulanger, Larose et Couturier, 2010; Tyler, 1996). La présence de ces écueils nécessite, d'une part, une réflexion critique sur les fondements de ces pratiques ainsi que sur le rapport qu'y entretiennent les chercheurs et, d'autre part, l'identification de tierces perspectives (Larose, Terrisse, Lenoir et Bédard, 2004; Oliver et Ostrofsky, 2007).

Nous assistons à l'émergence de conceptions alternatives du partenariat école-famillecommunauté s'articulant au construit d'écosystémie, tendance qui s'accompagne d'un questionnement sur les rapports aux fondements (De Gioia, 2009; Hughes et Greenhough, 2007; Hughes et Pollard, 2006; Price-Mitchell, 2009). Ces auteurs contextualisent le partenariat et ils en identifient des dynamiques socioculturelles sous-jacentes, en s'intéressant particulièrement à ce qui se joue à l'interface des systèmes. Sous cet angle, ils adoptent pour certaines considérations une perspective écosystémique d'orientations contextuelle et socioculturelle. En nous inspirant de ces auteurs, nous proposons d'inscrire notre lecture des pratiques collaboratives et de l'engagement parental au regard d'une telle logique.

\section{L'écosystémie dans une perspective contextuelle et socioculturelle}

Nous définissons les pratiques collaboratives et l'engagement parental au regard d'une perspective contextuelle et socioculturelle. Plus particulièrement, nous sollicitons les dynamic systems theory qui sont essentiellement caractérisées par le postulat d'une intégration dynamique entre les différentes composantes de l'écosystème (Smith et Thelen, 2003; Thelen, 2005; Witherington, 2007). Les systèmes sont considérés comme enchevêtrés et fusionnés entre eux. La configuration des liens entre les systèmes (mésosystème) se modifie et évolue en fonction d'un ensemble de dynamiques et de processus interactionnels et socioculturels qui leur donnent forme. Cette perspective théorique fournit un ensemble d'indices de définition de l'écosystème qui sont utiles pour conceptualiser et analyser les pratiques collaboratives, telles que nous les avons définies.

Les tenants des dynamic systems theory se sont davantage intéressés au développement de l'enfant qu'aux pratiques co-construites par les adultes exerçant une fonction éducative auprès de celui-ci. C'est en référence aux travaux d'Engeström (1987, 2001, 2009), s'inscrivant dans ce sillage théorique, que nous pouvons appliquer efficacement cette conception de l'écosystème à l'analyse de pratiques collaboratives. L'étude qu'il fait d'un ensemble de pratiques à base collective (entrepreneuriales, inter-organisationnelles, etc.) est fortement teintée aux plans dialectique, contextuel et socioculturel. Sa perspective est transposable à 
l'analyse des pratiques collaboratives et de l'engagement parental. Sans interpeller directement Engeström, Price-Mitchell (2009) fonde d'ailleurs sa lecture du partenariat école-famillecommunauté sur la dimension de boundary object qui est centrale dans les théories de l'activité et qui en reflète la perspective d'analyse dialectique des rapports socio-symboliques.

Pour Engeström, les activités des différents acteurs sont appelées à s'intégrer de manière dynamique pour former, au fil du temps, des pratiques collectives. Celles-ci prennent ancrage au plan culturel par le biais d'échanges dynamiques (dialectiques) et récurrents entre les acteurs. Nécessitant la confrontation des points de vue et la négociation de l'action (des objets, des finalités et des composantes de l'intervention), ces pratiques s'appuient sur un processus d'interculturalité. Celui-ci se réalise au sein d'interfaces culturelles, à la charnière des différents systèmes sociaux, et il aboutit à des synthèses constructives qui constituent la base d'émergence des pratiques.

Ce processus dynamique est médié ${ }^{12}$ par des outils d'ordre matériel (des dispositifs), relationnel et symbolique (Engeström, 2001). Ainsi, les représentations ${ }^{13}$ ou les discours des acteurs, les espaces relationnels ou les réseaux qui sont créés en cours d'action ainsi que les matériaux (des écrits, par exemple) utilisés dans le cadre de démarches collectives façonnent les pratiques et les conditions interactionnelles et socioculturelles dans lesquelles elles s'exercent. C'est par leur engagement que les acteurs contribuent à ces pratiques. Par l'usage, la mise en action et la construction d'outils de médiation, les partenaires cherchent à établir une forme de consensus en articulant les objets culturels dont ils sont respectivement vecteurs. Sous cet angle, les pratiques collectives se réalisent par le biais de l'engagement de l'ensemble des acteurs et elles constituent le produit intégrateur des activités et plus largement des cultures en « interaction ». L'engagement parental désigne alors, non pas un objet du discours ou des pratiques des professionnels, mais une composante ou une constituante centrale des pratiques collaboratives.

12. Engeström situe au niveau du mésosystème l'analyse des conditions d'interaction proximales, telles que documentées par Vygotsky. La zone proximale de développement correspond aux potentialités d'articulation et d'intégration horizontale (de synthèses) entre des systèmes ou des cultures et donc de développement de pratiques intégratrices par les acteurs. II parle d'expansion pour désigner ce processus. Sous cet angle, nous renvoyons à la médiation socioculturelle pour caractériser la dynamique socio-symbolique qui prend forme au sein d'interfaces et qui conduit à des formes de synthèses. Cette dynamique s'actualise par l'usage d'outils en contexte d'action par des acteurs.

13. Les représentations sociales, plus particulièrement, désignent des savoirs de sens commun co-construits par le biais d'échanges dynamiques entre les acteurs et par le biais de leurs pratiques (Marková, 2000). Elles s'organisent autour d'interactions et elles déterminent ces dernières. Elles prédisposent à l'action. Elles relèvent d'une logique contextuelle et dialectique. Afin de ne pas alourdir notre analyse en utilisant un trop grand nombre de construits fortement connotés théoriquement et épistémologiquement, nous recourrons au terme de représentation, construit générique qui renvoie à toute forme de pensée qui traduit un regard sur le monde et qui prédispose à l'action. Nous le situons dans une perspective sociale et autour d'une fonction de structuration de pratiques et d'interactions. Les représentations sont façonnées par ces dernières également. Nous considérons les représentations comme des outils sémantiques qui médiatisent le lien entre deux microsystèmes, comme l'école et la famille. Le mésosystème prend forme de manière directe, mais aussi indirecte par le biais d'interactions médiées. Les représentations des intervenants au regard desparents façonnent le lien écolefamille et déterminent les occasions d'engagement parental (De Gioia, 2009). 


\section{LE PROGRAMME FAMILLE, ÉCOLE, COMMUNAUTÉ, RÉUSSIR ENSEMBLE : TERRAIN D'ÉTUDE DE L'ÉCOSYSTÉMIE}

Le programme Famille, école, communauté, réussir ensemble (FECRE) constitue un terrain pertinent à l'étude de l'écosystémie, considérée comme une base de structuration des pratiques collaboratives ainsi que de l'engagement parental (Larose, Bédard, Couturier, Larivée, Lenoir, Lenoir et Terrisse, 2010). Dans le cadre de FECRE, ces pratiques s'appuient sur des structures soutenant de façon effective la mobilisation des acteurs autour de cibles communes. De plus, les intervenants qui s'y engagent ont comme obligation d'évaluer annuellement les effets des activités qu'ils exercent et de les redéfinir ou de les réajuster si elles sont considérées comme peu probantes ou s'ils veulent y apporter des modifications dans le but d'en potentialiser l'impact. Le programme est fondé sur une reconnaissance des compétences éducatives parentales de la part des professionnels et sur l'exploitation et le développement de ces dernières. Pour ces considérations, il fournit des conditions de structuration des liens sociaux et d'articulation entre diverses cultures en interaction (Boulanger et Larose, 2011sp). II peut alors constituer un terrain propice à la démonstration de l'actualisation d'une logique écosystémique d'orientations contextuelle et socioculturelle. C'est en définissant les deux principales structures du programme, soit les équipes locales et les plans de réussite, et en insistant sur les secondes, que nous pourrons le démontrer et en faire l'illustration.

Douze équipes locales ont été répertoriées en 2009 sur l'ensemble du territoire couvert par FECRE ${ }^{14}$. II s'agit de structures partenariales extérieures à l'école et composées de personnels scolaires et de professionnels intervenant dans le milieu. Les pratiques collaboratives sont définies, mises en œuvre et évaluées au sein des équipes locales. Contrairement à beaucoup de programmes, la mise en place de stratégies visant directement à recruter, à intéresser et à mobiliser les acteurs du milieu au sein et autour des équipes locales est particulièrement centrale (Larose, Bédard, Couturier, Larivée, Lenoir, Lenoir et Terrisse, 2010). Quelques parents, particulièrement ceux qui étaient fortement engagés à l'école avant l'implantation de FECRE, participent aux équipes locales. L'engagement parental s'exerce dans le cadre de plusieurs activités dont certaines sont planifiées, construites et co-animées par des parents de msef. Ces activités constituent des occasions d'interactions. Ces dernières tendent à se stabiliser dans le temps et donc à se réaliser de manière récurrente, ceci en raison du caractère récursif des démarches réalisées par les membres des équipes locales.

Les équipes locales sont animées par une ou un agent de développement dont le rôle est d'assurer la réalisation des plans de réussite et de soutenir la mobilisation de l'ensemble des acteurs sur une base active et continue. Quelques personnels enseignants assurent une présence stable au sein des équipes locales. L'agente ou l'agent de développement collabore avec les membres de l'équipe-école dans le cadre de la définition des moyens des plans de réussite. Elle ou il assure ainsi un relais et un transfert de l'information entre l'équipe locale et l'équipe-école. Les personnels enseignants qui s'engagent de manière récurrente dans le cadre des réunions des équipes locales ainsi que les directions d'école favorisent aussi cette articulation. La présence des agentes et des agents de développement à des tables territoriales

14. Rappelons qu'il a été implanté dans des écoles de msef. 
permet, par ailleurs, le rayonnement de FECRE dans le milieu. Ce sont les plans de réussite des écoles, définis, mis en œuvre et évalués par les membres des équipes locales, qui catalysent, matérialisent, donnent ancrage et permettent le vaste déploiement des pratiques collaboratives. C'est autour de ces dispositifs que sont les plans de réussite que sera axée notre analyse.

\section{Les plans de réussite : des dispositifs structurants fondés sur une logique écosystémique}

Avant de parler de ce qui caractérise les plans de réussite, nous traitons de la notion de dispositif. Ce dernier peut être défini comme une structure, une instance ou un ensemble de paramètres de nature structurelle et structurale qui organisent l'action et l'interaction sociale et qui sont façonnés et s'actualisent par et autour d'interactions stables ou de réseaux sociaux (Chambon et Proux, 1998; Foudriat et Immel, 2003). Pour ces auteurs, un dispositif de nature structurante soutient de façon effective la mobilisation et l'engagement d'acteurs sur une base collective. En tant que dispositifs structurants, les plans de réussite sont conçus dans une logique foncièrement écosystémique. Ils sont répartis en cinq sections, considérées comme des cibles globales et désignant les systèmes suivants : l'enfant, la famille, la classe, l'école et la communauté. Les intervenants mobilisés dans le cadre de FECRE visent à potentialiser l'effet de facteurs de protection. Dans une perspective écosystémique d'orientations contextuelle et socioculturelle, ces derniers sont considérés comme des ressources culturelles, relationnelles et symboliques qui sont construites par le biais d'échanges et d'interactions et dont le sens dépend du contexte dans lequel ils s'actualisent (Lerner, 2006). L'exploitation de ressources potentielles et leur développement dépendent des interactions qui s'exercent entre les acteurs. Les facteurs de protection sont une composante centrale des plans de réussite et ainsi des pratiques collaboratives et de l'engagement parental. Comme l'illustre le tableau 1 , trois facteurs (de protection) sont associés à chacune des cinq cibles (systèmes). II s'agit de déterminants que la documentation scientifique associe fortement à la réussite scolaire ou éducative (Lefebvre, Terrisse et Blain, 2003). 


\section{Tableau 1 : Répartition des facteurs en fonction des cibles ${ }^{15}$}

\begin{tabular}{|c|c|c|}
\hline Cible & Facteur & Description \\
\hline \multirow{3}{*}{ Cible 1 : L'ENFANT-ÉLÈVE } & Facteur 1 & Perception de sa propre compétence \\
\hline & Facteur 2 & Relations avec les pairs \\
\hline & Facteur 3 & Compétences en lecture et écriture \\
\hline \multirow{3}{*}{ Cible 2 : LA FAMILLE } & Facteur 4 & Revenu et scolarité \\
\hline & Facteur 5 & Implication des parents dans l'école \\
\hline & Facteur 6 & Place de la scolarisation en famille \\
\hline \multirow{3}{*}{ Cible 3 : LA CLASSE } & Facteur 7 & Perceptions et attitudes des enseignants et enseignantes \\
\hline & Facteur 8 & Choix pédagogique (pédagogie de la coopération) \\
\hline & Facteur 9 & Qualité de l'enseignement \\
\hline \multirow{3}{*}{$\underline{\text { Cible } 4 \text { : L'ÉCOLE }}$} & Facteur 10 & Direction et leadership \\
\hline & Facteur 11 & Services intrascolaires \\
\hline & Facteur 12 & Développement professionnel \\
\hline \multirow[b]{3}{*}{ Cible 5: LA COMMUNAUTÉ } & Facteur 13 & Quartier de résidence, voisinage \\
\hline & Facteur 14 & Mobilisation pour la scolarisation \\
\hline & Facteur 15 & Services extrascolaires \\
\hline
\end{tabular}

Pour chacune des cibles, les acteurs doivent minimalement déterminer un facteur à conserver tout au long de l'année scolaire en tant que ressource qu'ils cherchent à bonifier et dont ils tendent à potentialiser l'action dans l'environnement de l'enfant. Des objectifs, qui se traduisent sous forme de moyens (d'activités), sont identifiés pour chaque facteur. Pour chacun des facteurs, les moyens se répartissent en cinq catégories (ou niveaux), ceci afin d'inscrire le plan de réussite dans une logique écosystémique. Par exemple, le facteur 3 (compétence en lecture et en écriture) peut comporter les cinq moyens suivants: prendre note des livres préférés des enfants (enfant); distribuer des livres aux parents (famille); réaliser des activités de lecture en classe (classe); offrir de la formation professionnelle aux enseignant(e)s dans le domaine de la lecture/écriture (école); et visiter la bibliothèque de la municipalité avec les enfants de l'école (communauté).

Comme nous venons de l'illustrer, les moyens associés à chaque facteur doivent interpeller l'ensemble des cinq systèmes. C'est en ce sens que nous mentionnions que pour un facteur donné, les moyens se répartissent en cinq catégories ou niveaux. Les plans de réussite sont écosystémiques à la fois parce qu'ils se structurent autour des cibles que sont les systèmes et parce que, pour chacun des moyens, l'ensemble de ces systèmes est interpellé. Ils le sont en tant que cibles d'intervention et comme ressources à exploiter. Nous constatons alors comment deux modes de sollicitation et de mise en action des systèmes s'entrecroisent, pour se

15. Source : (Terrisse, Bédard et Hammani, 2005). 
potentialiser. Les plans de réussite sont ainsi organisés de façon à permettre la prise en compte de l'ensemble des déterminants de l'environnement de l'enfant et à favoriser une articulation entre les systèmes. Ainsi, le fait de solliciter la bibliothèque comme ressource dans le but de renforcer l'effet d'un facteur de protection qui concerne directement l'enfant (ses compétences en lecture et en écriture par exemple) permet une intégration de l'ensemble des composantes de son écosystème. Les services de la bibliothèque seront interpellés pour soutenir le potentiel d'action d'autres facteurs et d'autres cibles. L'ensemble des ressources de l'environnement pourra alors être mis à profit et agir à différents niveaux.

\section{Des tendances majeures illustrant l'application d'une logique écosystémique d'orientations contextuelle et socioculturelle au partenariat école-famille-communauté}

Le programme FECRE a fait l'objet d'une évaluation sur une période de trois ans, soit entre 2006 et 2009 (Larose, Bédard, Couturier, Larivée, Lenoir, Lenoir et Terrisse, 2010). Sans faire une description de la méthodologie de cette recherche évaluative ni une description systématique des résultats, nous présentons quelques-unes des conclusions qui se dégagent de cette étude et qui permettent d'illustrer l'application d'une logique écosystémique d'orientations contextuelle et socioculturelle aux pratiques collaboratives ainsi qu'à l'engagement parental.

Mentionnons que nous nous appuyons de façon particulière sur une analyse lexicométrique du discours des acteurs qui se sont engagés dans le programme (Leimdorfer et Salem, 1995). Nous avons utilisé antérieurement une procédure similaire pour investiguer diverses dimensions des dynamiques relationnelles créées ou soutenues par ce programme (Boulanger, Larose et Couturier, 2010; Larose, Boulanger, Couturier, Bédard et Larivée, 2009; Larose, Couturier et Boulanger, 2007). Par le biais de cette méthode d'analyse, nous avons pu situer sur un plan graphique le contenu d'entrevues semi-dirigées individuelles et de groupe et ainsi identifier des noyaux de sens commun qui reflètent le discours partagé d'un ensemble de catégories d'acteurs. Pour une définition précise de ce qui caractérise la statistique textuelle ou lexicométrique, le lecteur se réfèrera à l'excellent ouvrage introductoire de Lebart et Salem $(1994)^{16}$.

À l'issue de la démarche évaluative, nous pouvons notamment énoncer les constats suivants :

- les agentes et les agents de développement ont fortement contribué à la mobilisation des acteurs et ils représentent la mémoire collective des pratiques exercées;

- le recours à des structures de mobilisation a grandement assuré la stabilité de la démarche ainsi que son évaluation;

16. L'ouvrage est maintenant disponible gratuitement en ligne [http://ses.telecom-paristech.fr/lebart/ST.html.] 
- l'inscription du programme dans une logique écosystémique a favorisé cette mobilisation, a permis le développement d'un ensemble de ressources, notamment dans la communauté, et elle a soutenu l'engagement des parents à titre de partenaires;

- les intervenants ont progressivement modifié les attitudes qu'ils portaient au regard des parents, en considérant ceux-ci de plus en plus comme des acteurs centraux au plan des apprentissages de l'enfant et en reconnaissant leurs compétences éducatives;

- et la mise en œuvre du programme sur une longue période de temps a permis la construction collective de savoirs et de pratiques ainsi que l'ancrage de ces dernières dans les milieux de pratique.

Ces résultats font ressortir les opportunités qu'offre le recours à une perspective écosystémique, non seulement comme grille de lecture de la réalité sociale (écosystème) de l'enfant, mais aussi comme orientation méthodologique déterminant les conditions de mise en œuvre d'un partenariat école-famille-communauté. Les agentes et les agents de développement considèrent que l'écosystémie exerce une fonction d'organisation et de structuration des liens entre les acteurs. Plus particulièrement, elle oriente la mobilisation de ceux-ci autour d'échanges constructifs et de dynamiques d'intégration horizontale entre un ensemble d'objets culturels, en l'occurrence les facteurs de protection qui sont une composante centrale des plans de réussite. Parce qu'elles sont potentialisées par une action collective complexe de la part des acteurs, ces ressources tendent à se développer de manière synergique. En témoignent, par exemple, non seulement l'amélioration des services offerts par la bibliothèque du milieu, mais aussi leur usage concomitant par plusieurs acteurs et pour différentes visées. La bibliothèque désigne un lieu d'interactions entre les parents et leurs enfants, un espace de réseautage entre les familles et une ressource utilisée par l'institution scolaire notamment (dans le cadre de l'animation d'ateliers à l'école par les bibliothécaires). II s'agit surtout d'une zone d'interface favorisant la création de liens entre l'ensemble des acteurs (parents, personnels enseignants, intervenants du milieu) qui s'y côtoient.

La bibliothèque, mais aussi d'autres espaces intermédiaires tels que l'école ou différents réseaux informels ont permis l'articulation croisée des différentes activités des plans de réussite. Par exemple, les intervenants des centres d'éducation des adultes ont pu recruter des parents intéressés à s'engager dans une démarche de formation, lors d'activités d'accès public large comme la rentrée scolaire. Des services de gardiennage, offerts par les éducatrices et les éducateurs de centres de la petite enfance de divers quartiers, ont aussi permis aux parents de s'engager dans différents comités.

$\mathrm{Si}$, le plus souvent, les parents ne font pas directement partie des équipes locales, ils sont partie prenante des pratiques collaboratives qui se déploient en son centre et à sa périphérie. Les diverses catégories de professionnels interagissent avec eux dans le cadre d'activités collectives telles que la rentrée scolaire, mais aussi dans leur quotidien. La communication 
entre les personnels enseignants et les parents tend progressivement à s'établir sur une base bidirectionnelle et régulière. Les parents vont souvent initier la communication. Ils s'engagent aussi activement dans le cadre des activités qui leur sont destinées et contribuent souvent à l'élaboration ou la réalisation d'ateliers. Ils exercent régulièrement un rôle d'animateur en classe, surtout lors des dernières années d'implantation du programme.

Les représentations des différentes catégories d'intervenants exercent une fonction de soutien par rapport à l'engagement parental. Comme nous le mentionnions, elles constituent un outil de médiation de l'interaction entre les acteurs et plus largement du lien école-famille (mésosystème ${ }^{17}$ ). Rappelons aussi qu'elles s'appuient sur les échanges ou les interactions et qu'elles prédisposent à l'action. L'ensemble des intervenants reconnaît progressivement les gains d'engager activement les parents et ils les considèrent de plus en plus compétents. Ils désignent l'engagement parental comme une action nécessaire au partenariat et à la réussite éducative de l'enfant. Les agentes et les agents de développement et en partie les directions d'école trouvent important d'établir un dialogue ouvert avec les parents et que ceux-ci puissent négocier avec les professionnels la nature des apprentissages de l'enfant. Un certain nombre de personnels enseignants considère, en fin de démarche, que la culture familiale et en partie celle du milieu communautaire sont à la base des acquis de l'enfant et des apprentissages qu'il réalise, notamment à l'école. Certains personnels enseignants ajustent leurs pratiques pédagogiques en tenant compte de la culture familiale, par exemple des différents usages de l'écrit à la maison. Au sein des équipes locales, mais aussi dans différents milieux tels que l'école, les intervenants véhiculent les représentations et les discours qu'ils adoptent. Par le biais de leurs échanges avec des tiers, ils promeuvent la culture familiale. Celle-ci devient progressivement une composante centrale des plans de réussite et des pratiques collaboratives.

Ces dernières se sont fortement stabilisées et elles ont pris ancrage dans la culture de certains des milieux de pratique interpellés, notamment en raison de la dynamique d'action des représentations qui y sont véhiculées. Le caractère structurant des plans de réussite, leur fondement écosystémique, mais aussi la fonction fédératrice des équipes locales ont permis d'assurer la stabilité des démarches, de consolider les pratiques, mais surtout de favoriser l'appropriation de ces dernières dans chacun des milieux. Plusieurs indices attestent du maintien des plans de réussite et plus particulièrement des pratiques collaboratives un an après la fin de l'implantation du programme. De plus, les directions d'école et les agentes et agents de développement affirment qu'ils continueront à promouvoir la « logique FECRE » qui teinte les discours des acteurs et qui oriente les pratiques collaboratives. Les réseaux créés à l'issue de la démarche et les dynamiques interactionnelles qui se sont déployées (le lien avec les parents en particulier) tendent aussi à perdurer.

17. Rappelons que les représentations sociales façonnent de façon indirecte le lien école-famille. Sous cet angle, l'engagement parental s'exerce non seulement par le biais des interactions entre les parents et les professionnels, mais aussi par le biais de l'action médiatrice des représentations. Ces dernières étant véhiculées par le biais des échanges des intervenants des équipes locales, elles influencent les plans de réussite et les pratiques. La reconnaissance des compétences éducatives parentales par ces intervenants favorise l'intégration, à ces échanges et à ces pratiques, de diverses composantes de la culture familiale. 
Les éléments qui viennent d'être mentionnés reflètent l'application de grands principes d'une logique écosystémique d'orientations contextuelle et socioculturelle, telle que nous l'avons décrite. Essentiellement, nous pouvons résumer le contenu qui vient d'être présenté par cinq grandes tendances:

- Le caractère structurant des plans de réussite fondés sur une logique écosystémique;

- La construction de pratiques collaboratives autour de dynamiques interactionnelles et socioculturelles (les représentations notamment) émergentes;

- La mise en œuvre d'un processus d'interculturalité dont les objets renvoient aux activités des acteurs et aux facteurs de protection;

- L'actualisation de pratiques interprofessionnelles soutenant le développement de ressources;

- L'engagement actif des parents dans le cadre des pratiques collaboratives.

Les pratiques collaboratives s'inscrivent clairement dans un registre partenarial, dans la mesure où elles se construisent autour d'interactions dynamiques et stables entre les acteurs professionnels et non professionnels et qu'elles s'appuient et découlent sur la formation de consensus entre ceux-ci. Ceci reflète une représentation intégrative du mésosystème qui se construit et prend forme autour d'espaces de fusion (des synthèses) et qui s'actualise sur une base à la fois contextuelle et socioculturelle. En tant qu'indices de mise en œuvre des pratiques collaboratives, les plans de réussite ont favorisé la médiation entre les cultures en présence. En particulier, les différentes activités exercées par les intervenants et par les parents ont pu s'articuler et s'intégrer autour de zones relationnelles émergentes, pensons à la bibliothèque qui a constitué un espace de rencontre et de médiation culturelle.

Les zones d'interface générées à l'issue de la démarche caractérisent le partenariat et elles ont permis le développement de ressources (facteurs de protection). La construction et la diffusion de représentations reflètent l'actualisation d'un processus d'interculturalité, surtout dans la mesure où les intervenants se représentent la culture familiale en tant que ressource à intégrer à leurs pratiques quotidiennes. L'engagement actif des parents dans le cadre des pratiques collaboratives traduit la création et le partage de savoirs ou, plus largement, d'objets culturels constitués à la charnière des systèmes. En témoigne la dynamique de production et d'actualisation des représentations des intervenants, telle que décrite préalablement. 


\section{Conclusion}

L'usage de l'écosystémie en tant que métathéorie a permis d'identifier des cas de figure du partenariat école-famille-communauté. Ayant insisté sur la perspective mécaniste, le continuum présenté laisse place au positionnement d'un ensemble d'autres tendances intermédiaires. Évoquons le modèle d'Epstein, qui peut s'inscrire dans une perspective organiste-mécaniste par la promotion qui y est faite d'un rapprochement entre systèmes en fonction d'un modèle scolaire et en référence aux principes de la cohérence et de la continuité (Vincent et Tomlinson, 1997). En croisant les fondements et les conditions de la pratique et en tenant compte de la composante socioculturelle, la perspective que nous proposons permet de réfléchir au lien école-famille-communauté sous des angles variés, celui de l'interaction, de l'engagement, des dynamiques socioculturelles et de l'articulation d'objets culturels. Ce que nous suggérons peut permettre d'englober, de systématiser et de compléter plusieurs tentatives de conceptualisation (par le biais de typologies), en particulier celles d'auteurs comme Swap (1990), lequel définit l'engagement en fonction de la nature du rôle parental.

La perspective contextuelle et socioculturelle proposée va en partie dans le sens des travaux effectués par plusieurs auteurs dans le champ de la littéracie (Cairney, 1997, 2000, 2001; Fleer et Williams-Kennedy, 2001). En recourant aux théories de l'activité d'Engeström, nous pouvons contribuer en partie à systématiser les dynamiques de fusion ou de synthèse entre les systèmes à l'égard desquelles les auteurs entretiennent généralement un rapport assez flou. Notre analyse mériterait d'être enrichie par l'intégration du concept de croyance épistémique que Schommer-Aikins (2004) situe dans une perspective écosystémique convergente à celle que nous avons suggérée. En effet, nous avons peu identifié le statut des savoirs des parents tel qu'attribué par les professionnels, ni même leur propre représentation de l'interaction et plus largement du mésosystème. Néanmoins, bien que perfectible, cette analyse s'avère opératoire dans le cadre de ses limites conceptuelles comme le démontre le bref exemple d'application aux dynamiques engendrées par le programme FECRE réalisé dans ces pages. Notre lecture de l'écosystémie et de ses fondements peut-elle s'avérer un analyseur efficace de tierces dynamiques relationnelles? Au lecteur d'en juger. 
Dany Boulanger

Étudiant au doctorat en éducation

Université de Sherbrooke

Maîtrise en travail social Baccalauréat en psychologie

François Larose

Professeur titulaire en enseignement au préscolaire et au primaire

Université de Sherbrooke

Doctorat en éducation

Serge J. Larivée

Professeur agrégé

Département de psychopédagogie et d'andragogie

Université de Montréal

Doctorat en sciences de l'éducation

Yves Couturier

Professeur titulaire

Service social, Université de Sherbrooke

Doctorat en sciences humaines appliquées

Corinne Mérini

Maître de conférences

Professeur titulaire

Sciences et techniques des activités physiques et sportives

IUFM de Versailles, France

Doctorat en sciences de l'éducation

François Blain Maîtrise en gestion de projets Université du Québec à Montréal Personne-ressource en réussite scolaire en milieu défavorisé et en mobilisation des communautés.

Valérie Cusson

Étudiante à la maîtrise en sciences cliniques

Université de Sherbrooke Doctorat en médecine dentaire (D.M.D.)

Daniel Moreau

Étudiant au doctorat en éducation

Université de Sherbrooke

Maîtrise en histoire

Baccalauréat en histoire

Naomi Grenier

Étudiante à la maîtrise en sciences de l'éducation

Université de Sherbrooke

Baccalauréat en psychologie (B.A.) 


\section{RÉFÉRENCES BIBLIOGRAPHIQUES}

Albee, G.W. (1982). «Preventing psychopathology and promoting human potential », American Psychologist, 37(9), p. 1043-1050.

Amatea Ellens, S. et A. D. Sherrard Peter (1994). "The ecosystemic view: A choice of lenses », Journal of Mental Health Counselling, 16(1), p. 6-21.

Baltes, P. B. (1987). « Theoretical propositions of life-span developmental psychology: On the dynamics between growth and decline », Developmental Psychology, 23(5), p. 611-626.

Baum, H. S. (2002). «The community approach to school-community partnerships: Challenges and possibilities », communication présentée à l'Annual Meeting of the American Educational Research Association, La Nouvelle-Orléans, 1-5 avril.

Boethel, M. (2003). Diversity: School, Family and Community Connections. Annual synthesis 2003, Austin (TX), National Center for Family and Community Connections with Schools/Southwest Educational Development Laboratory.

Boudreault, P., J.-C. Kalubi et A. C. Moreau (2000). «Compétences des parents et évaluation du développement de leur enfant : de l'appropriation à l'autodétermination », Les Cahiers de l'Actif, $\mathrm{n}^{\text {os }}$ 288-291, p. 165-178.

Boudreault, P., J.-C. Kalubi, L. Sorel, P. Beaupré et J.-M. Bouchard (1998). «Recherches sur l'appropriation des savoirs et des savoir-faire entre parents et intervenants », dans S. L. Éthier et J. Alary, Comprendre la famille, Québec, Presses de l'Université du Québec, p. 316-330.

Boulanger, D. et F. Larose (2011sp). «Building a school-family-community partnership based on a structuring device: Success plans », communication au congrès de l'European Association for Reseach on Learning and Instruction (EARLI), Exeter (G.-B.), 30 août au 3 septembre.

Boulanger, D., F. Larose et Y. Couturier (2010). «La logique déficitaire en intervention sociale auprès des parents : les pratiques professionnelles et les représentations sociales », Nouvelles pratiques sociales, 23(1), p. 152-176.

Branco, A. (1996). "The "ecological" approach: When labels suggest similarities beyond shared basic concepts in psychology », dans J. Tudge, M. Shanahan et J. Valsiner (dir.), Comparisons in Human Development: Understanding time and context, New York, Cambridge University Press, p. 304-317.

Breitborde, M.-L. et L. B. Swiniarski (2002). « Family education and community power: New structures for new visions in the educational village », Educational Studies, 28(3), p. 305-318.

Bronfenbrenner, U. (1974). "Developmental research, public policy, and the ecology of childhood», Child Development, 45(1), p. 1-5.

Bronfenbrenner, U. (1977). «Toward an experimental ecology of human development », American Psychologist, 32, p. 513-531.

Bronfenbrenner, U. (1979). The Ecology of Human Development: Experiments by nature and design, Cambridge, Harvard University Press.

Bronfenbrenner, U. (1986). «Ecology of the family as a context for human Development: Research perspectives », Developmental Psychology, n²2, p. 723-742. 
Bronfenbrenner, U. (2005). Making Human Beings Human: Bioecological perspectives on human development, Thousand Oaks (CA), Sage.

Cairney, T. H. (1997). « Acknowledging diversity in home literacy practices: Moving towards partnership with parents », Early Child Development and Care, 127-128, p. 61-73.

Cairney, T. H. (2000). « Beyond the classroom walls: The rediscovery of the family and community as partners in education », Educational Review, 52, p. 163-174.

Cairney, T. H. (2001). «Bridging home and school literacy: In search of transformative approaches to curriculum », Early Child Development and Care, 172(2), p. 153-172.

Cantillon, D. (2006). «Community social organization, parents, and peers as mediators of perceived neighborhood block characteristics on delinquent and prosocial activities », American Journal of Community Psychology, 37(1-2), p. 111-127.

Chambon, A. et M. Proux (1988). «Zones d'éducation prioritaires: un changement social en éducation? », Revue française de pédagogie, 83, p. 31-38.

Christenson, S. L. (2003). "The family-school partnership: An opportunity to promote the learning competence of all students », School Psychology Quarterly, 18(4), p. 454-482.

Christenson, S. L. et S. M. Sheridan (2001). Schools and Families: Creating essential connections for learning, New York, Guilford Press.

Cole, M. (1999). «Culture in development », dans M. H. Bornstein et M. E. Lamb (dir.), Developmental Psychology: An advanced textbook, Mahwah (NJ), Erlbaum, p. 73-124.

Couturier, Y., F. Larose et J. Bédard (2009). « Continuités et discontinuités de l'intervention éducative et de l'éducation socioéducative en milieu scolaire défavorisé », Les sciences de l'éducation pour l'ère nouvelle, 42(4), p. 93-116.

Davies, S. et N. Guppy (1997). « Focus on English-speaking democracies. Globalization and educational reforms in Anglo-American democracies », Comparative Education Review, 41(4).

De Gioia, K. (2009). «Parent and staff expectations for continuity of home practices in the child care setting for families with diverse cultural backgrounds », Australian Journal of Early Childhood, 34(3), p. 9-17.

Deschesnes, M., Martin, C. et A. J. Hill (2003). « Comprehensive approaches to school health promotion: How to achieve broader implementation? », Health Promotion International, 18(4), p. 387-396.

Deslandes, R. et L. Lafortune (2001). «La collaboration école-famille dans l'apprentissage des mathématiques selon la perception des adolescents », Revue des sciences de l'éducation, 27(3), p. 649-669.

Deslandes, R., E. Royer, P. Potvin et D. Leclerc (1999). « Patterns of home and school partnership for general and special education students at the secondary level », Exceptional Children, 65(4), p. 496506.

Driessen, G., F. Smit et P. Sleegers (2005). «Parental involvement and educational achievement », British Educational Research Journal, 31(4), p. 509-532.

Eccles, J. S. et R. D. Harold (1993). «Parent-school involvement during the early adolescent years », Teacher's College Record, 94, p. 568-587. 
Edwards, A. et J. Warin (1999). «Parental involvement in raising the achievement of primary school pupils: Why bother? », Oxford Review of Education, 25(3), p. 325-341.

Engeström, Y. (1987). Learning by Expanding: An activity-theoretical approach to developmental research, Helsinki, Orienta-Konsultit Oy.

Engeström, Y. (2001). « Expansive learning at work: Toward an activity theoretical reconceptualization », Journal of Education and Work, 14(1), p. 133-156.

Engeström, Y. (2009) «From learning environments and implementation to activity systems and expansive learning », Action: An International Journal of Human Activity Theory, $\mathrm{n}^{\circ}$ 2, p. 17-33.

Epstein, J. K. (1987). «Toward a theory of family-school connections: Teacher practices and parent involvement », dans K. Hurrelmann, F. Kaufmann et F. Losel (dir.), Social Intervention: Potential and constraints, New York, De Gruyter, p. 121-136.

Epstein, J. L. (1995). « School/family/community partnerships: Caring for the children we share », Phi Delta Kapan, 76, p. 701-711.

Epstein, J. L. (2005). «A case study of the Partnership Schools Comprehensive School Reform (CSR) model », Elementary School Journal, 106(2), p. 151-170.

Fan, X. T. et M. Chen (2001). «Parental involvement and students' academic achievement: A metaanalysis », Educational Psychology Review, 13, p. 1-22.

Ferlazzo, L. (2011). « Involvement or engagement? », Educational Leadership, mai, p. 10-14.

Fitzgerald, L. M. et A. Goncü (1993). «Parent involvement in urban early childhood education : A Vygotskian approach », dans R. Stuart (dir.), Advances in Early Education and Day Care, 5: Perspectives on developmentally appropriate practices, Greenwich, JAI Press, p. 197-212.

Fleer, M. et D. Williams-Kennedy (2001). Building Bridges: Literacy development in young indigenous children, Canberra, Australian Early Childhood Association Inc.

Foudriat, M. et B. Immel (2003). «Temporalités des processus de coopération entre acteurs dans les changements organisationnels et dispositifs d'interventions sociologiques », dans C. Blanchard-Laville et D. Fablet (dir.), Théoriser les pratiques professionnelles, Paris, L’Harmattan, p. 127-145.

Garbarino, J. et D. Sherman (1980). «High-risk neighborhoods and high-risk families: The human ecology of maltreatment », Child Development, 51, p. 188-198.

Gibbs, J. C. (1979). "The meaning of ecologically oriented inquiry in contemporary psychology », American Psychologist, 34(2), p. 127-140.

Gibson, J. J. (1962). « Observations on active touch », Psychological Review, 69(6), p. 477-491.

Gibson, E. J. (1967). «On the proper meaning of the term "stimulus" », Psychological Review, 74(6), p. 533-534.

Gouvernement du Québec (2003a). Stratégie de mise en œuvre 2003-2006 de l'Entente de complémentarité des services entre le réseau de la santé et des services sociaux et le réseau de l'éducation, Québec, Ministère de la Santé et des Services sociaux et Ministère de l'Éducation, du Loisir et du Sport. 
Gouvernement du Québec (2003b). Le plaisir de réussir se construit avec mon entourage. Cadre de référence, Québec, Ministère de la Santé et des Services sociaux et Ministère de l'Éducation. Document téléaccessible à l'adresse suivante :

[http://www.mels.gouv.qc.ca/dfga/politique/fecre/pdf/cadre_fecre.pdf].

Gouvernement du Québec (2004). Rapprocher les familles et l'école primaire, Québec, Ministère de l'Éducation, du Loisir et du Sport. Document téléaccessible à l'adresse suivante :

[http://www.mels.gouv.qc.ca/].

Gouvernement du Québec (2008). Deux réseaux, un objectif : le développement des jeunes, Québec, Ministère de l'Éducation, du Loisir et du Sport. Document téléaccessible à l'adresse suivante :

[http://www.mels.gouv.qc.ca/sections/publications/publications/EPEPS/Formation_jeunes/Adaptation_scolai re/2Reseai1ObjectifProtocoleEntente_f.pdf].

Greenberg, M. T., M. E. Feinberg, S. Meyer-Chilenski, R. L. Spoth et C. Redmond (2007). « Community and team member factors that influence the early phase functioning of community prevention teams: the PROSPER project », Journal of Primary Prevention, 28, p. 485-504.

Henderson, A. T. et K. L. Mapp (2002). A New Wave of Evidence: The impact of school, family, and community connections on student achievement, Austin (TX), Southwest Educational Development Laboratory. [http://www.sedl.org/pubs/catalog/items/fam33.html].

Henrich, C. C. (2006). "Context in action: Implications for the study of children and adolescents », Journal of Clinical Psychology, 62(9), p. 1083-1096.

Hughes, M. et P. Greenhough (2007). " "We do it a different way at my school" », dans S. Watson et P. Winbourne (dir.), New Directions for Situated Cognition in Mathematics Education, Université d'Oxford, London South Bank University, p. 129-152.

Hughes, M. et A. Pollard (2006). « Home-school knowledge exchange in context », Educational Review, 58(4), p. 385-395.

Jeynes, W. H. (2005). «A meta-analysis of the relation of parental involvement to urban elementary school student academic achievement », Urban Education, 40(3), p. 237-269.

Jordan, C., E. Orozco et A. Averett (2001). Emerging Issues in School, Family and Community Connections, Austin (TX), National Center for Family and Community Connections with Schools/ Southwest Educational Development Laboratory.

Keyes, C. R. (2002). «A way of thinking about parent/teacher partnerships for teachers », International Journal of Early Years Education, 10(3), p. 177-191.

Kelly, J. G. (1966). «Ecological constraints on mental health services », American Psychologist, 21(6), p. 535-539.

Kondrat, M. E. (2002). «Actor-centered social work: Re-visioning "person-in-environment" through a critical theory lens », Social Work, 47(4), p. 435-448.

Landry, S. J., K. Levin, D. Rowe et M. Nickelson (2010). « Enabling collaborative work across different communities of practice through boundary objects: Field studies in air traffic management », International Journal of Human-Computer Interaction, 26(1), p. 75-93.

Larivée, S., J.-C. Kalubi et B. Terrisse (2006). «La collaboration école-famille-communauté en contexte d'inclusion : entre obstacles, risques et facteurs de réussite », Revue des sciences de l'éducation, 32(15), p. 525-543. 
Larose, F., J. Bédard, Y. Couturier, S. J. Larivée, A. Lenoir, Y. Lenoir et B. Terrisse (2010). Étude évaluative des impacts du programme "Famille, école, communauté, réussir ensemble » (FECRE) sur la création de communautés éducatives soutenant la persévérance et la réussite scolaire d'élèves "à risque » au primaire, Sherbrooke, Université de Sherbrooke. Rapport de la recherche FQRSC \# 2007-EC-118198. [http://www.fqrsc.gouv.qc.ca/upload/editeur/RF_FrancoisLarose(1).pdf].

Larose, F., D. Boulanger, Y. Couturier, J. Bédard et S. J. Larivée (2009). « As representações sociais como analisador da emergência de uma nova profissão em educação : o caso dos agentes de desenvolvimento no Québec », Revista da FAEEBA - Educação e Contemporaneidade, 18(32), p. 129-146.

Larose, F., Y. Couturier et D. Boulanger (2007). "Conditions et enjeux de l'actualisation de l'interdisciplinarité professionnelle pour les formations initiales à l'enseignement et à l'exercice du travail social au Québec », Les dossiers des sciences de l'éducation, 17, p. 77-93.

Larose, F., Y. Lenoir, Y. Couturier et J. Bédard (2011sp). «L'essor de l'interdisciplinarité professionnelle au temps des réformes à la faveur de l'émergence d'une zone d'intégration des pratiques d'intervention éducatives et socio-éducatives », Revue des sciences de l'éducation, 35(3).

Larose, F., B. Terrisse, Y. Lenoir et J. Bédard (2004). « Approche écosystémique et fondements de l'intervention éducative précoce en milieux socio-économiquement faibles. Les conditions de la résilience scolaire », Brock Education, 13(2), p. 56-80.

Lawson, M. A. (2003). « School-family relations in context », Urban Education, 39(1), p. 77-134.

Lebart, L. et A. Salem (1994). Statistique textuelle, Paris, Dunod.

Lefebvre, M. L., B. Terrisse et F. Blain (2003). Les facteurs-clés de succès liés à la réussite scolaire au primaire. Revue commentée de la littérature récente. Document disponible à l'adresse suivante : [http://www.mels.gouv.qc.ca/dfga/politique/fecre/facteurs/pdf/revuecommentee.pdf].

Leimdorfer, F. et A. Salem (1995). «Usages de la lexicométrie en analyse de discours », Cahier des sciences humaines, 31(1), p. 131-143.

Lemay, L., R. Giguère et A. Marchand (2008). «La médiation partenariale en contexte intersectoriel: intervention sociale en émergence, codéveloppement professionnel et recherche ", Sociétés et jeunesses en difficulté, 6 . Document téléaccessible à l'adresse suivante :

[http://sejed.revues.org/document4002.html.]

Lenoir, Y. (2005). «Le "Rapport Parent" ou le début de l'ancrage de l'école québécoise dans la logique anglophone nord-américaine, Canadian Journal of Education / Revue Canadienne de l'éducation », 28(4), p. 638-668.

Lerner, R. M. (1998). «Theories of human development: Contemporary perspectives », dans R. M. Lerner (dir.), Theoretical Models of Human Development, vol. 1 de W. Damon et R. M. Lerner (dir.), Handbook of Child Psychology, $5^{\mathrm{e}}$ éd., New York, Wiley, p. 1-24.

Lerner, R. M. (2004). « Diversity in individual $\leftrightarrow$ context relations as the basis for positive development across the life span: A developmental systems perspective for theory, research, and application ", Research in Human Development, 1(4), p. 327-346.

Lerner, R. M. (2006). « Resilience as an attribute of the developmental system: Comments on the papers of Professors Masten and Wachs », dans B. M. Lester, A. Masten et B. McEwen (dir.), Resilience in Children. Annals of the New York Academy of Sciences, New York, New York Academy of Sciences. 
Lerner, R. M., A. M. Freund, I. De Stefanis et T. Habermas (2001). « Understanding developmental regulation in adolescence: The use of the selection, optimization, and compensation model », Human Development, 44, p. 29-50.

Levasseur, M., L. Richard, L. Gauvin et E. Raymond (2010). «Inventory and analysis of definitions of social participation found in the aging literature: Proposed taxonomy of social activities », Social Science and Medicine, 71, p. 2141-2149.

Lott, B. (2001). «Low-income parents and the public schools », Journal of Social Issues, 57(2), p. 247259.

Luhmann, N. (2006). « System as difference », Organization, 13(1), p. 37-57.

Marková, I. (2000). «Amédée or how to get rid of it: Social representations from a dialogical perspective », Culture and Psychology, 6(4), p. 419-460.

McClenaghan, P. (2000). «Social capital: Exploring the theoretical foundations of community development », British Educational Research Journal, 26(5), p. 555-582.

McGarth, W. H. (2007). «Ambivalent partners: Power, trust, and partnership in relationships between mothers and teachers in a full-time child care center », Teachers College Record, 109(6), p. 14011422.

Mérini, C. (1995). «Entre savoir scolaire et pratiques sociales : le partenariat à l'école », Spirale - Revue de recherches en éducation, $\mathrm{n}^{\circ} 16$, p. 169-184.

Mérini, C. (2001). «Le partenariat: histoire et essai de définition », dans Actes de la Journée nationale de l'OZP, 5 mai.

Meuret, D. (2006). « Equity and efficiency of compulsory schooling: Is it necessary to choose and if so on what grounds? », Prospects, 36(4), p. 389-410.

Mickan, S., S. J. Hoffman et L. Nasmith (2010). « Collaborative practice in a global health context: Common themes from developed and developing countries », Journal of Interprofessional Care, 24(5), p. $492-502$.

Moll, L. (1993). «Community-mediated educational practices », communication présentée à l'American Educational Research Association Annual Conference, Atlanta (GA), 12-16 avril.

Moll, L. C., C. Amanti, D. Neff et N. Gonzalez (1992). «Funds of knowledge for teaching: Using a qualitative approach to connect homes and classrooms », Theory Into Practice, 31(2), p. 132-141.

Morris, E. K. (1997). "Some reflections on contextualism, mechanism, and behaviour analysis », Psychological Record, 47(4), p. 529-542.

Nagda, B. A., M. L. McCoy et M. H. Barrett (2006). « Mix It Up: Crossing social boundaries as a pathway to youth civic engagement », National Civic Review, 95(1), p. 47-56.

Nichols, S. (2007). "Parental involvement in supporting children with learning difficulties », dans W. Louden et al. (dir.), Mapping the Territory. Primary students with learning difficulties: literacy and numeracy, Canberra, Department of Education, Training and Youth Affairs.

Oliver, L. E. et R. Ostrofsky (2007). «The ecological paradigm of mind and its implications for psychotherapy », Review of General Psychology, 11(1), p. 1-11. 
Overton, W. F. (1998). « Developmental psychology: Philosophy, concepts, and methodology », dans R. M. Lerner (dir.), Theoretical Models of Human Development, vol. 1 de W. Damon et R. M. Lerner (dir.), Handbook of Child Psychology, $6^{\mathrm{e}}$ éd., New York, John Wiley and Sons, p. 107-188.

Overton, W. F. (2007). «A coherent metatheory for dynamic systems: Relational organicismcontextualism », Human Development, 50, p. 154-159.

Overton, W. F. et M. D. Ennis (2006). « Cognitive-developmental and behaviour-analytic theories: Evolving into complementary », Human Development, 49, p. 143-172.

Pepper, S. C. (1942). World Hypotheses: A study in evidence, Berkeley (CA), University of California Press.

Powell, D. R. (2001). « Visions and realities of achieving partnership: Parent-teacher relationships at the turn of the century », dans M. von Cranach, W. Doise et G. Mugny (dir.), Social Representations and the Social Bases of Knowledge, Lewiston (NY), Hogrefe and Huber, p. 333-357.

Price-Mitchell, M. (2009). « Boundary dynamics: Implications for building parent-school partnerships », The School Community Journal, 19(2), p. 9-26.

Reese, H. W. et W. F. Overton (1970). « Models of development and theories of development », dans L. R. Goulet et R. B. Baltes (dir.), Life-Span Developmental Psychology: Research and theory, New York, Academic Press, p. 116-145.

Riegel, K. F. (1976). «The dialectics of human development », American Psychologist, 31, p. 689-700.

Sameroff, A. J. (1983). «Developmental systems: Contexts and evolution », dans P. Mussen (dir.), Handbook of child psychology, 1, p. 237-294.

Seginer, R. (2006). "Parents' educational involvement: A developmental ecology perspective», Parenting: Science and Practice, 6(1), p. 1-48.

Schommer-Aikins, M. (2004). «Explaining the epistemological belief system: Introducing the embedded systemic model and coordinated research approach », Educational Psychologist, 39(1), p. 19-29.

Selsky, J. W. (1991). "Lessons in community development: An activist approach to stimulating interorganizational collaboration », Journal of Applied Behavioral Science, 27(1), p. 91-115.

Sheldon, S. B et F. L. Van Voorhis (2004). «Partnership programs in U.S. schools: Their development and relationship to family involvement outcomes", School Effectiveness and School Improvement, 15(2), p. 125-148.

Smith, L. B. et E. Thelen (2003). « Development as a dynamic system », TRENDS in Cognitive Sciences, $7(8)$, p. 343-348.

Sontag, J. C. (1996). «Toward a comprehensive theoretical framework for disability research: Bronfenbrenner revisited », The Journal of Special Education, 30(3), p. 319-344.

Souto-Manning, M. et K. Swick (2006). «Teachers' beliefs about parent involvement: Rethinking our family involvement paradigm », Early Childhood Education Journal, 34(2), p. 187-193.

Swap, S. M. (1990). «Comparing three philosophies of home-school collaboration », Equity and Choice, 6(3), p. 9-19. 
Sykes, G. (2001). «Home-school agreements: A tool for parental control or for partnership? », Educational Psychology in Practice, 17(3), p. 273-286.

Terrisse, B. et F. Larose (2003). « La formation des intervenants socio-éducatifs auprès de l'enfant et de la famille : une nouvelle profession, de nouvelles compétences », Revue internationale de l'éducation familiale, 7(1), p. 5-7.

Terrisse, B., J. Bédard et A Hammami (2005). Le projet « Famille, école, communauté : réussir ensemble (FECRE)». Recension et analyse commentée de projets d'interventions socioéducatives en milieu socioéconomiquement faible (éducation préscolaire et enseignement primaire), DGFA, Ministère de l'Éducation du Québec et Montréal (QC), Groupe de recherche en adaptation scolaire et sociale (GREASS), DEFS, Université du Québec à Montréal.

Tessier, R. (dir.) (1989). Pour un paradigme écologique, Montréal, HMH.

Tessier, R. et G. M. Tarabulsy (dir.) (1996). Le modèle écologique dans l'étude du développement de l'enfant, Sainte-Foy, Presses de l'Université du Québec.

Thelen, E. (2005). « Dynamic system theory and the complexity of change », Psychoanalytic Dialogues, 15(2), p. 255-283.

Thomas, S. C. (1996). «A sociological perspective on contextualism », Journal of Counselling and Development, 74, p. 529-536.

Tudge, J. R. H., J. Gray et D. Hogan (1997). «Ecological perspectives in human development: A comparison of Gibson and Bronfenbrenner », dans J. Tudge, M. Shanahan et J. Valsiner (dir.), Comparisons in Human Development: Understanding time and context, New York, Cambridge University Press, p. 72-105.

Tyler, K. (1996). «Systems thinking and ecosystemic psychology », Educational Psychology, 16(1), p. 21-34.

Ungar, M. (2002). «A deeper, more social ecological social work practice », Social Service Review, 76(3), p. 480-497.

Valsiner, J. (2003). " Culture and its transfer: Way of creating general knowledge through the study of cultural particulars », Online Readings in Psychology and Culture, 2, no 11.

[http://orpc.iaccp.org/index.php].

Valsiner, J. (2009). "Cultural psychology today: Innovations and oversights », Culture and Psychology, 15(1), p. 5-39.

Vincent, C. (1993). «Community participation? The establishment of "City's Parents" Centre », British Education Research Journal, 19(3), p. 227-242.

Vincent, C. et J. Martin (2002). «Class, culture and agency: Researching parental voice », Discourse: Studies in the Cultural Politics of Education, 23(1), p. 109-128.

Vincent, C. et S. Tomlinson (1997). "Home-school relationships: "The swarming of disciplinary mechanisms"? », British Educational Research Journal, 23(3), p. 361-377.

Wapner, S. et J. Demick (1998). « Developmental analysis: A holistic, developmental, systems-oriented perspective », dans R. M. Lerner (dir.), Theoretical Models of Human Development, vol. 1 de W. Damon et R. M. Lerner (dir.), Handbook of Child Psychology, $5^{\mathrm{e}}$ éd., New York, Wiley, p. 761-805. 
Weerts, D. J. et L. R. Sandmann (2010). «Community engagement and boundary-spanning roles at research universities », The Journal of Higher Education, 81(6), p. 632-157.

Wilgus, G. (2005). " "If you carry him around all the time at home, he expects one of us to carry him around all day here and there are only TWO of us !" Parents', teachers', and administrators' beliefs about the parent's role in the infant/toddler center », Journal of Early Childhood Teacher Education, $n^{0} 26$, p. 259-273.

Witherington, D. C. (2007). «The dynamic systems approach as metatheory for developmental psychology », Human Development, 50, p. 127-153.

Wong, W. C. (2001). «Co-constructing the personal space-time totality: Listening to the dialogue of Vigotsky, Lewin, Bronfenbrenner, and Stern », Journal for the Theory and Social Behaviour, 31(4), p. 365-382. 